\title{
CAN'T INTUBATE ANAPLASTIC THYROID CARCINOMA - A CASE REPORT
}

\author{
Sigdel B, Dubey T, KC Neeraj, Nepali R, Maharjan R, Sah K, Upadhyaya K \\ Department of Otolaryngology and Head and Neck surgery, \\ Gandaki Medical College, Pokhara, Nepal
}

\begin{abstract}
Female, 66 years old with a chief complain of shortness of breath and lump over her neck since 6 month. Neck USG showed enlarged right lobe of thyroid gland with hyperechoic nodules. FNAC confirmed anaplastic carcinoma of thyroid. patient underwent chemotherapy using doxorubicin as agent of choice. She had difficulty in breathing and she kept on high flow of oxygen, even after she had stridor and planned for tracheostomy. MRI revealed heterogeneously enhancing mass $(9.6 * 6.5 * 10.5 \mathrm{~cm})$ replacing right lobe of thyroid, and extending superiorly till the level carotid bifurcation and inferiorly superior mediastinum. Lesion is partially encasing trachea and laryngeal airway, posteriorly extending into prevertebral space and anteriorly invading strap muscles and sternocleidomastoid muscle. Now it become very difficult stage. She transfers to ICU. intubation tried but failed. As mass was just anterior to trachea and difficult to do tracheostomy without intubation. all hope failed and she put continue to high flow of oxygen.
\end{abstract}

Key Words: Anaplastic, Thyroid, Carcinoma, malignant, thyroid cancer

\section{INTRODUCTION}

Anaplastic thyroid cancer is a malignant tumor originated from a follicle cell with the highest incidence rate of sixth and seventh decade of life. Anaplastic lesion constitutes less than $5 \%$ of thyroid cancer but it does $50 \%$ of thyroid cancerrelated deaths. ${ }^{1,2}$ This type of thyroid cancer is one of the highest mortality rate of malignancy with a life expectancy less than 6 months. ${ }^{3,4}$ The etiology of this malignancy is currently unknown, The numerous molecular alteration detected in anaplastic thyroid cancer. ${ }^{5}$

This type of cancer can cause severe compression of the structures in the neck leading to shortness of breath, dysphagia, and vocal cord paralysis. ${ }^{6}$ Tracheal invasion is present in $25 \%$ at the time of presentation. This is why many patients with anaplastic thyroid cancer will need a tracheostomy. ${ }^{7}$

\section{Corresponding Author}

Dr.Brihaspati Sigdel, HOD and Associate Professor, Department of Otolaryngology and Head and Neck surgery, Gandaki Medical College, Phone no, Office: 061-538595, Mobile: 9779856030090
When the diagnosis is made, most of the patient has a regional lymph enlargement, and around $40 \%$ already has distant metastases especially to lung, bone, and brain. ${ }^{8}$ An intensive and multimodal approach, which includes surgical resection and radiotherapy and combined adjuvant chemotherapy, improve local and survival in some patients with regionally confined ATC. ${ }^{9,10}$

\section{Case Report}

A female patient of age 66 years old came with a chief complaint of shortness of breath since 1 week (on 27/08/2019 at Gandaki Medical College emergency department). Shortness of breath was making the patient unable to do her daily activity. Patient also complaint of having a lump over her neck since 7 month ago. It was said the size of the lump was increasing progressively day by day. The lump had a hard consistency, fixed position, and there was no pain. There was no fever. she also complaint of cough which was productive in character. she was diagnosed anaplastic carcinoma of thyroid last year in the month January 2019 
at another center as per FNAC report. she was a known case of Chronic obstructive pulmonary disease (COPD) under aerosol, hypothyroidism and hypertension. There was no family history with the same complaint as above listed.

In physical examination, vital sign stable, with a general appearance of severe illness, patient was fully alert, dyspneic, blood pressure $90 / 70 \mathrm{mmHg}$, axilla temperature $97.60 \mathrm{~F}$, with pulse rate 114beats/ minute, respiration rate $24 \mathrm{breath} /$ minute, oxygen saturation $98 \%$. On eye examination there was no pale conjunctiva and normal pupillary reflex. Neck examination there was a thyroid gland enlargement with a size approximately $8-10 \mathrm{~cm}$, hard, nodular ,fixed, and no pain on palpation. There was a marked lymph node enlargement on the right side of the neck and right submandibular region. On chest auscultation, bilateral wheeze present and cardiac examination reveals normal 1 st and 2 nd heart sounds. Abdominal examination revealed there was no abnormality, with a normal bowel

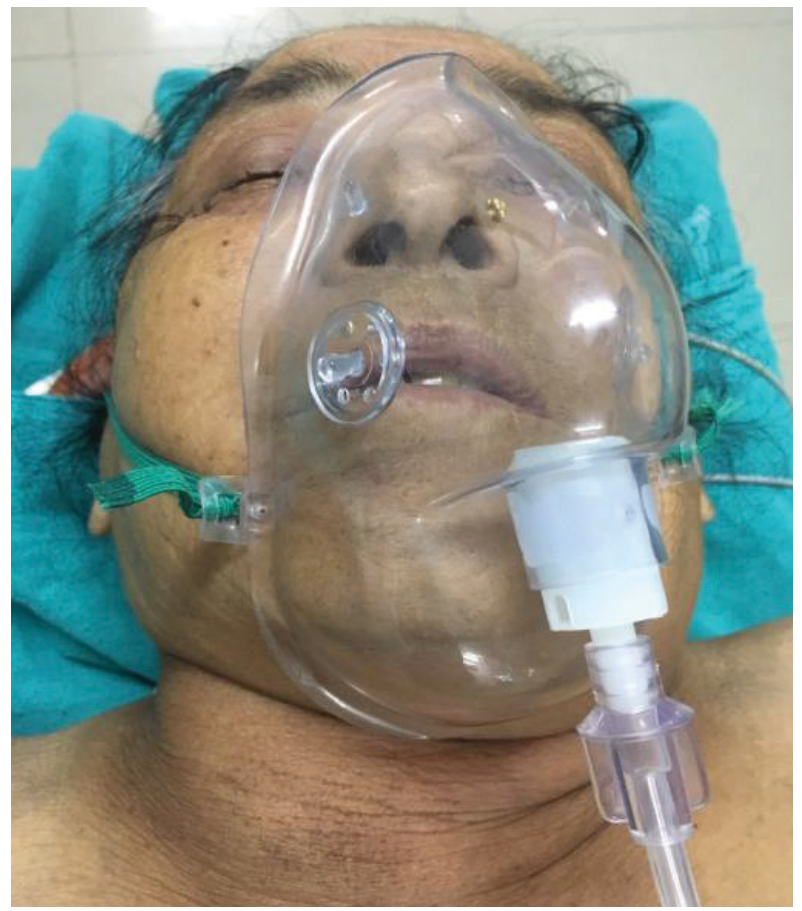

Figure 1. showing patient coming with respiratory distress with stridor on high flow oxygen
On complete blood count examination revealed a white blood cell $16.2 \times 10^{3} / \mu \mathrm{L}$, haemoglobin $13.8 \mathrm{gram} / \mathrm{dl}$, platelets $2,25,000$. Blood chemistry panel with result urea $26 \mathrm{mg} / \mathrm{dl}$, creatinine $0.9 \mathrm{mg} /$ $\mathrm{dl}$, RBS $133 \mathrm{mg} / \mathrm{dl}$. Thyroid function test revealed a hypothyroid condition with level of TSH 58.27 $(0.30-5.00) \mu \mathrm{IU} / \mathrm{mL}$, FT3 $1.19 \mathrm{pg} / \mathrm{ml}$, FT4 $0.57 \mathrm{ng} / \mathrm{dl}$.

Previous Record of patient Neck USG shows enlarged right lobe of thyroid gland with hyperechoic nodules. FNAC confirmed anaplastic carcinoma of thyroid.

Patient was treated with 5 cycles of chemotherapy and 25 days of radiation therapy.

Patient had stridor so planned for tracheostomy. before doing tracheostomy, neck evaluation done with ultrasonography and MRI.

MRI revealed large heterogeneously enhancing mass $(9.6 * 6.5 * 10.5 \mathrm{~cm})$ replacing right lobe of thyroid, and extending superiorly till the level carotid bifurcation and inferiorly superior mediastinum. lesion is encasing right common carotid artery and proximal portion of subclavian artery. However vessels show normal flow void. Right internal jugular vein not seen. Lesion is partially encasing trachea and laryngeal airway, posteriorly extending into prevertebral space and anteriorly invading strap muscles and sternocleidomastoid muscle. Left lobe of thyroid gland shows heterogenous enhancement. Small nodular enhancing lesion within right occipital region-likely metastasis in brain.

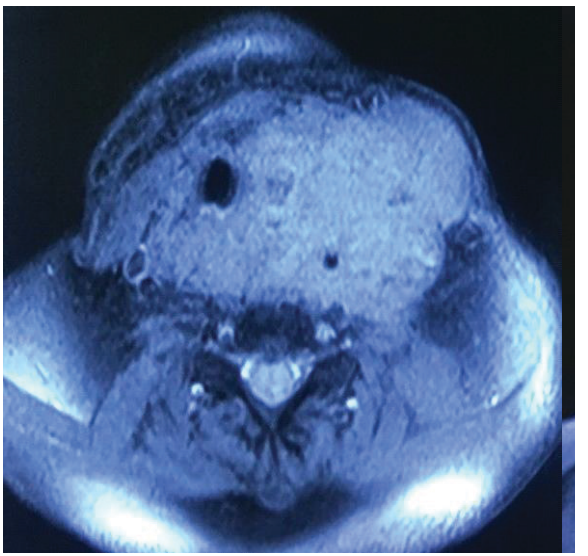

A 

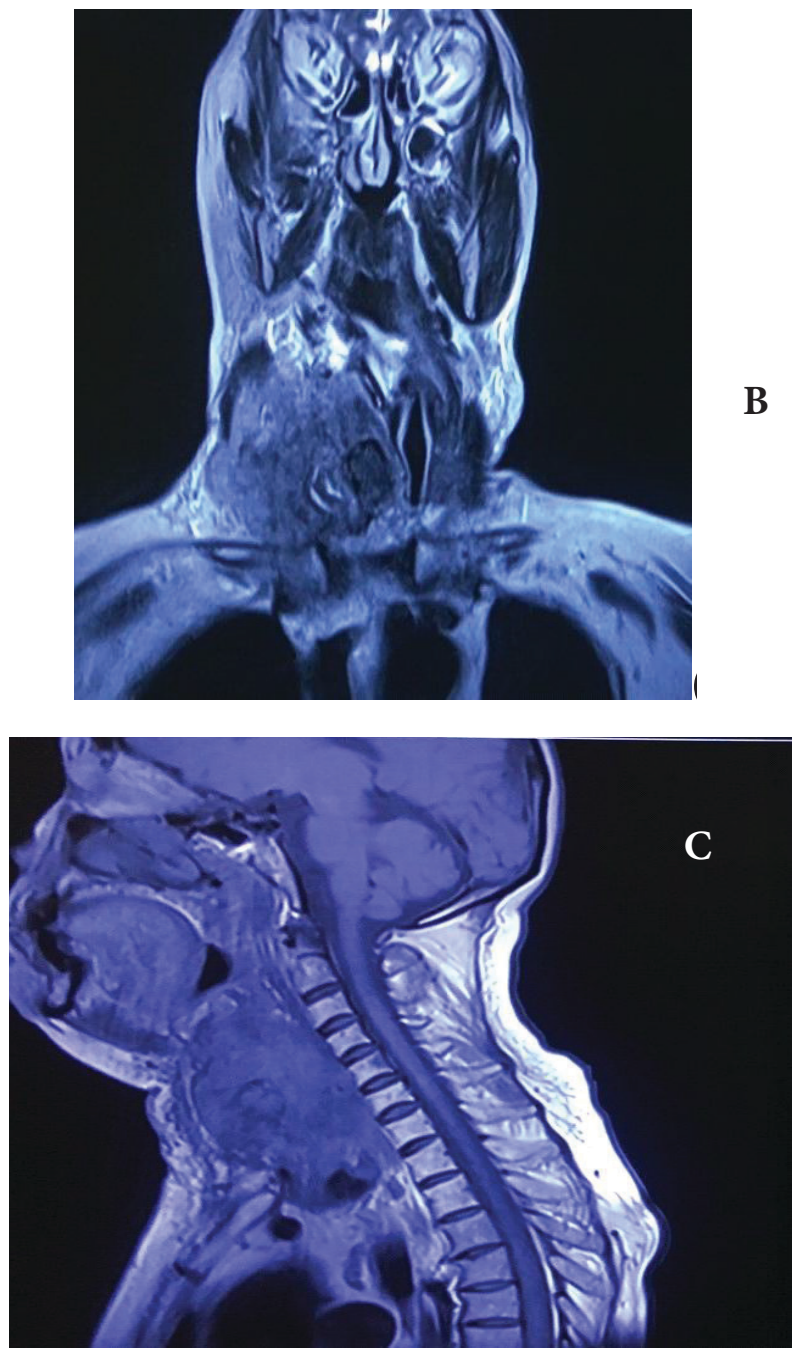

Figure 2. MRI A-axial, B-coronal-sagittal showing extension of the thyroid mass

With the clinical manifestation and also supported by the additional lab result, it was concluded that the patient had anaplastic thyroid cancer with difficult airway. She was kept in ICU under Oxygen therapy@61/min via face mask. She was planned for tracheostomy under GA to relieve stridor. Anesthetist consultation done and patient transferred to OT and intubation failed. 3 cycles of chemotherapy with doxorubicin was given. patient kept with high flow oxygen and patient expired after 2 weeks.

\section{Discussion}

Anaplastic thyroid cancer is one of the most aggressive solid tumors in humans, with an average survival of 6 months. The 1-year and 10-year survival rates are estimated to be $10-20 \%$ and less than $5 \%$. Although only $1-3 \%$ of all thyroid cancers, but anaplastic thyroid cancer contributes to $14-49 \%$ of deaths from thyroid cancer. ${ }^{2}$ Anaplastic thyroid cancer is more common in women than in males and peak occurs in the sixth and seventh decades of life. The exact etiology of anaplastic thyroid cancer remains unknown. But it is thought to be due to the interaction between genetic factors, and the environment. Genetic alteration with multiple dedifferentiation occur in ATC. ${ }^{11}$ Risk factors for anaplastic thyroid cancer include goiter history, iodine deficiency, radiation, and female sex.

Clinical manifestations of anaplastic thyroid cancers can develop rapidly by invading surrounding local tissues and metastases into distant organs. Locally, anaplastic thyroid cancer shows rapid anterior neck enlargement accompanied by dysphagia (40\%), sound change or hoarseness $(40 \%)$, and stridor (24\%). Regional symptoms include enlarged lymph nodes $(54 \%)$, and neck pain $(26 \%){ }^{8}$ Systemic symptoms include anorexia, weight loss and shortness of breath with pulmonary metastases. On physical examination, the thyroid enlargement measured $8-10 \mathrm{~cm}$ in size, palpable with solid consistency. In patients with thyroid nodules, serum TSH levels should be examined. If serum TSH is low, then a radioiodine examination is performed. If serum TSH is normal or elevated, continued with ultrasound (ultrasound) ultrasonography and fine needle aspiration based on clinical and ultrasound results. High serum TSH levels are associated with an increased risk of malignancy from thyroid nodules. In the case, the thyroid function check results indicate the patient has hypothyroidism in which the patient's serum TSH level increases.

Ultrasound examination, CTscan and histopathology confirm the thyroid cancer. Ultrasound provides us with information about extensions, nodular 
structures and invasive thyroid disease. ${ }^{12,13}$ In this case, thyroid USG shows enlarged right lobe of thyroid gland with heteroechoic nodules.

Routine imaging of all patients should be performed to evaluate the spread of the disease locally and to exclude the presence of distant metastasis. ${ }^{13}$ Anaplastic thyroid cancer may invade structures in the middle, lateral, and mediastinal neck by direct tumor invasion or by lymphatic invasion. In this tumor invaded trachea and larynx causing airway narrowing.

On histopathological examination of anaplastic thyroid cancer, we can find a squamous, spindle cells and giant cells. Often encountered with high mitotic activity, extensive necrosis. In this case, we found tumor cells arranged in clusters and singly scattered cells have high N:C ratio with moderate to abundant amount of cytoplasm and highly pleomorphic vesicular nuclei. Few of the cells are spindle shaped with elongated nuclei. Background shows necrosis and hemorrhage.

Most patients with anaplastic thyroid cancer do not require pneumatic airway intervention unless the patient has acute respiratory disturbance or severe stridor. Tracheostomy in anaplastic thyroid cancer patients is performed in the event of lifethreatening asphyxia. Tracheostomy should be avoided where possible. However, if the patient has severe respiratory problems, the patient should be tracheostomized. ${ }^{14,15}$ In this case, the patient experiences an episode of airway obstruction with a narrowing of the tracheal lumen due to a tumor causing the patient to tightness and stridor. Patients was planned to do a tracheostomy procedure, but unfortunately it was failed in our cases.

\section{Conclusion}

A case of a 66 years old woman diagnosed with anaplastic thyroid cancer had been reported and died. Anaplastic thyroid cancer is a very rare cancer and has a poor prognosis. The important information such as clinical manifestations, physical examination, and support is necessary to diagnose and administer proper management of anaplastic thyroid cancer patients.

\section{References}

1. Cornett WR, Sharma AK, Day TA, Richardson MS, Hoda RS, van Heerden JA, et al. Anaplastic thyroid carcinoma: an overview. Current oncology reports. 2007;9(2):152-8.

2. Besic N, Auersperg M, Us-Krasovec M, Golouh R, Frkovic-Grazio S, Vodnik A. Effect of primary treatment on survival in anaplastic thyroid carcinoma. European Journal of Surgical Oncology (EJSO). 2001;27(3):260-4.

3. Pasieka JL. Anaplastic thyroid cancer. Current opinion in oncology. 2003;15(1):78-83.

4. Chiacchio S, Lorenzoni A, Boni G, Rubello D, Elisei R, Mariani G. Anaplastic thyroid cancer: prevalence, diagnosis and treatment. Minerva endocrinologica. 2008;33(4):341-57.

5. Ragazzi M, Ciarrocchi A, Sancisi V, Gandolfi G, Bisagni A, Piana S. Update on anaplastic thyroid carcinoma: morphological, molecular, and genetic features of the most aggressive thyroid cancer. International journal of endocrinology. 2014;2014.

6. Cooper DS, Doherty GM, Haugen BR, Kloos RT, Lee SL, Mandel SJ, et al. Revised American Thyroid Association management guidelines for patients with thyroid nodules and differentiated thyroid cancer: the American Thyroid Association (ATA) guidelines taskforce on thyroid nodules and differentiated thyroid cancer. Thyroid. 2009;19(11):1167-214.

7. Xu J, Liao Z, Li JJ, Wu XF, Zhuang SM. The Role of Tracheostomy in Anaplastic Thyroid Carcinoma. World J Oncol. 2015;6(1):262-4.

8. Molinaro E, Romei C, Biagini A, Sabini E, Agate L, Mazzeo S, et al. Anaplastic thyroid carcinoma: from clinicopathology to genetics and advanced therapies. Nat Rev Endocrinol. 2017;13(11):644-60.

9. Saini S, Tulla K, Maker AV, Burman KD, Prabhakar BS. Therapeutic advances in anaplastic thyroid cancer: a current perspective. 